ÉGYPTE monde arabe

\section{Égypte/Monde arabe}

12 | 2015

Evolution des systèmes médiatiques après les révoltes arabes

\title{
Les graffitis de la rue Mohammed Mahmoud. Dialogisme et dispositifs médiatiques
}

\section{Zoé Carle et François Huguet}

\section{(2) OpenEdition}

\section{Journals}

Édition électronique

URL : https://journals.openedition.org/ema/3449

DOI : 10.4000/ema.3449

ISSN : 2090-7273

Éditeur

CEDEJ - Centre d'études et de documentation économiques juridiques et sociales

Édition imprimée

Date de publication : 25 mars 2015

Pagination : 149-176

ISBN : 9782905838858

ISSN : 1110-5097

Référence électronique

Zoé Carle et François Huguet, « Les graffitis de la rue Mohammed Mahmoud. Dialogisme et dispositifs médiatiques », Égypte/Monde arabe [En ligne], 12 | 2015, mis en ligne le 25 mars 2015, consulté le 07 juillet 2022. URL : http://journals.openedition.org/ema/3449; DOI : https://doi.org/10.4000/ema.3449 


\section{Zoé Carle \& François Huguet}

\section{RÉSUMÉ / ABSTRACT}

Partant d'un double point de vue, à la fois empirique et numérique, cet article observe la relation entre l'émergence spectaculaire de graffitis dans l'espace urbain du Caire et l'essor des médias sociaux dans le développement d'une nouvelle culture contestataire en Egypte. Nous concentrons notre étude sur les graffitis de la rue Mohamed Mahmoud pour observer comment cet espace urbain particulier devient un espace médiatique et symbolique par le biais de son investissement par les réseaux sociaux. La rue en elle-même est l'espace privilégié d'une nouvelle forme d'expression, le graffiti, une zone d'écriture en acte que nous pouvons définir selon la terminologie de Béatrice Fraenkel comme un sanctuaire d'écriture (Fraenkel, 2002) qui nous semble être le laboratoire de nouvelles pratiques d'action et d'expression collective au sein de la jeunesse égyptienne. En effet, la ville du Caire a connu récemment l'émergence d'un nouvel ordre graphique dans lequel les citoyens inscrivent leurs actes de résistance par le biais de représentations picturales ou scripturales. Ces inscriptions doivent être prises en compte avec leurs représentations numériques qui les reproduisent ou les discutent sur Internet. Ensemble, elles semblent avoir initié dans le monde arabe contemporain un mécanisme complexe : une succession d'actes articulés les uns aux autres qui produisent de nouvelles situations de communication et d'échange (Gonzalez-Quijano, 2011). Ce travail vise ainsi à évaluer l'importance de la rue Mohamed Mahmoud comme lieu d'exposition de formes dialogiques d'écriture dans le changement socioculturel que connaît une certaine partie des publics égyptiens et plus largement arabes.

MOTS CLÉS :

Graffitis, espace publique, nouveaux médias, réseaux sociaux, cyberactivisme. 
From two interrelated points of view, both empirical and digital, this work examines the relationship between the emergence of massive graffiti in Cairo, the rise of social media and the intensification of a contemporary protest culture in Egypt. Indeed, we have seen the emergence on the walls of the Egyptian capital of a new graphic order in which citizens engage themselves in acts of resistance through urban writings and pictorial representations. This "writing event" (Fraenkel, 2002), at work in Cairo since the beginning of the revolution in January 2011 is the symbol of a renewed relationship with the Egyptian public space. This work focuses on the murals of Mohamed Mahmoud street in Cairo and the transformation of this public space into a symbolic place. The street is the privileged space of a new form of political expression, a writing-in-action area, and thus it constitutes a good example of the shift in the collective actions among Egyptian youth who are familiar with ICTs. This work will attempt to articulate a vision of Mohamed Mahmoud street as an exhibition space for dialogical forms of writing, but also as the birthplace of a socio-cultural shift in a certain sector of Egyptian and Arab audiences: a space able to shape a new relationship with the media.

\section{KEYWORDS:}

Graffiti, Public Space, New Media, Social Networks, Digital Activism.

ZOÉ CARLE est doctorante en littérature comparée au sein du CERC (Centre d'Etudes et de Recherches Comparatistes) de l'université Paris 3. Ses recherches portent sur les liens entre littérature populaire et littérature d'avant-garde, en particulier sur la poétique des slogans révolutionnaires, en tant qu'outil de création d'un imaginaire révolutionnaire dans certains moments historiques et en tant que modèle de création en littérature. Elle étudie en particulier le cas de l'Egypte contemporaine et la création artistique révolutionnaire depuis janvier 2011.

- Carle, Z. (2014), "Force de frappe: les slogans entre séduction et méfiance ", Revue L'Intermède, Paris [en ligne].

- Carle, Z. and Huguet, F. (2013), "Les graffitis de la rue Mohammed Mahmoud, Palimpseste de la révolution égyptienne? » (à paraitre - Presses Universitaires de Rouen et du Havre)

zoe.carle@gmail.com 
FRANÇOIS HUGUET est doctorant au département Sciences Économiques et Sociales de Telecom ParisTech (UMR CNRS LTCI - CoDesign Lab \& Media Studies). Ses recherches concernent les nouvelles formes d'infrastructures de télécommunication et les enjeux sociopolitiques des architectures informatiques décentralisées. Sa thèse s'appuie notamment sur une ethnographie du déploiement de réseaux sans fil communautaires à Détroit et prend place au croisement de la sociologie des sciences et des techniques et des sciences de l'information et de la communication.

Il travaille parallèlement sur les scripts de la place Tahrir et leurs « reflets» numériques.

- Huguet, F. (2014), «Taïwan manifeste en peer-to-peer», Médiapart.fr, 3 avril 2014. [en ligne]

- Huguet, F. (2014), « Recoudre la ville avec du sans fil : Dispositifs d'encapacitation des publics et discours d'infrastructures participatives », in GIS Démocratie et Participation, Actes des 3èmes Journées Doctorales.

- Huguet, F. (2013), « Pour une ethnographie des réseaux sans fil communautaires : Implémenter des technologies décentralisées pour agir contre la crise à Détroit », Revue Urbanités, Paris. [en ligne]

- Carle, Z. and Huguet, F. (2013), «Les graffitis de la rue Mohammed Mahmoud, Palimpseste de la révolution égyptienne? » (à paraitre - Presses Universitaires de Rouen et du Havre).

- Huguet, F. (2013), «Tahrir et ses écritures dans le miroir » in «Les médias font-ils les révolutions?»(dir. F. Daghmi, F. Toumi, A. Amsidder), L'Harmattan - Communication et Civilisations, Paris.

Gentès, A. and Huguet, F. (2012), «Les alternatives aux réseaux sociaux : I'architecture distribuée et le design de média », dans Réseaux sociaux. Culture politique et ingénierie des réseaux sociaux (éd. Stiegler, B.), IRI FYP, Paris.

francois.huguet@telecom-paristech.fr 


\section{LES GRAFFITIS DE LA RUE MOHAMMED \\ MAHMOUD, DIALOGISME ET DISPOSITIFS MÉDIATIQUES}

"En réalité et en pratique, le vrai message, c'est le médium lui-même, c'est-à-dire, tout simplement, que les effets d'un médium sur l'individu ou sur la société dépendent du changement d'échelle que produit chaque nouvelle technologie, chaque prolongement de nous-mêmes, dans notre vie ». Pour comprendre les médias, Seuil, Paris, p. 37.

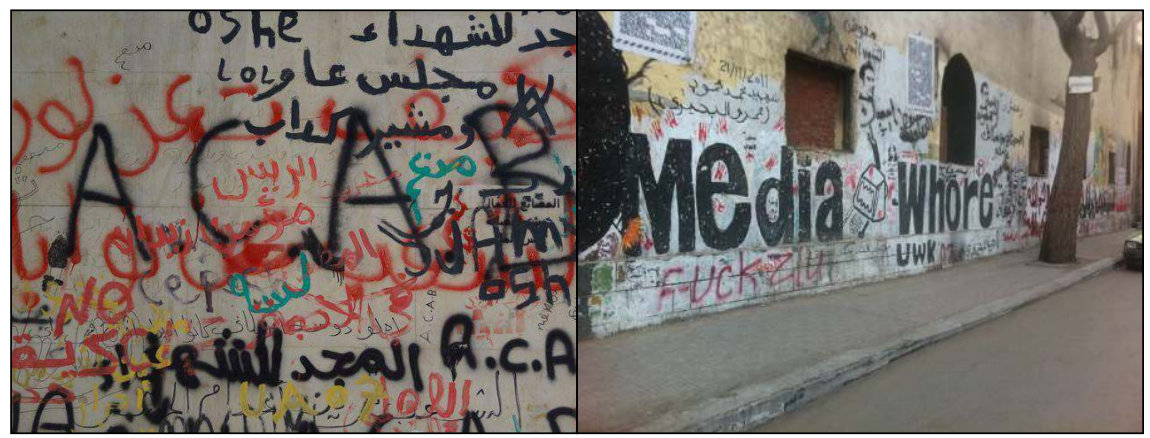

Rue Mohammed Mahmoud, Le Caire (Égypte), janvier et novembre 2013, crédits photos: Zoé Carle, François Huguet. 


\section{DE LA CIRCULATION DES ÉCRITURES EXPOSÉES}

De nombreux travaux ont abordés les graffitis égyptiens, forme la plus visible d'un "art révolutionnaire" apparu avec les soulèvements de janvier 2011; néanmoins, un aspect particulier de ces productions culturelles reste peu étudié, celui du rapport qu'elles entretiennent avec les médias numériques. À partir d'une double perspective disciplinaire, entre anthropologie de l'écriture et sciences de l'information et de la communication, nous souhaitons nous interroger sur les liens privilégiés qu'entretiennent ces objets, à mi-chemin entre œuvres artistiques et gestes politiques, avec l'usage des réseaux sociaux numériques. Nous tâchons ainsi d'analyser les cheminements de leurs projets politiques en recomposant une partie de l'histoire de ces œuvres, de leurs trajectoires sociales et poétiques, afin de montrer de quelle manière des valeurs spécifiques ont été intégrées aux principes de création et de circulation de ce qui se présente comme un nouveau dispositif médiatique. Nous pensons en effet, à la suite d'Yves Jeanneret (2008) et de son travail sur la circulation dans la société de ce qu'il nomme des "êtres culturels" que nos savoirs, nos valeurs morales, nos catégories politiques et nos expériences esthétiques ne peuvent se transmettre que dans un cheminement à travers les carrefours de la vie sociale en se métamorphosant, en produisant du nouveau et en se chargeant de valeur. D'un point de vue communicationnel, les graffitis de la rue Mohammed Mahmoud (MM) n'échappent pas à ces modelages sociaux ni à ces recompositions. Bien au contraire, ils nous semblent liés de manière intrinsèque aux modes de circulation et de discussion des différentes significations qu'ils véhiculent à la fois in situ, dans cette célèbre artère du centre-ville du Caire, adjacente à la non moins fameuse place Tahrir, épicentre du soulèvement égyptien de 2011, et ex situ $^{1}$, sur les pages Facebook regroupant des photographies des graffitis et des différentes couches physiques et sémiotiques dont se composent les murs de la rue. Ces graffitis sont des êtres culturels qui modélisent une parole politique particulière, une voix d'opposition aux discours diffusés par les médias officiels traditionnels (TV, journaux, radios). Ils reflètent ce qui se discute et s'écrit sur d'autres murs, pourtant moins matériels, ceux des réseaux sociaux numériques.

Si I'histoire de l'apparition d'"arabités numériques" a été étudiée par Yves Gonzalez-Quijano ${ }^{2}$ et si l'émergence spectaculaire de nouvelles formes artistiques dans la ville du Caire a fait l'objet d'une extraordinaire couverture médiatique depuis les trois dernières années - en témoigne

1. Nous pourrions également parler de "significations-communications urbi et orbi" à la place de in et ex situ.

2. Gonzalez-Quijano, 2012. 
la parution d'au moins six ouvrages portant uniquement sur les graffitis et un grand nombre $d^{\prime}$ articles universitaires ou journalistiques ${ }^{3}$ - les liens privilégiés entre ces créations et les mondes du numérique ont été peu explorés. Nous souhaitons examiner ici, en croisant une approche anthropologique de terrain, se basant sur des entretiens avec des artistes graffitis et des relevés photographiques, et une approche moins traditionnelle d'observation de la circulation des œuvres sur Internet, les conséquences de cette intrication étroite d'une forme d'art et de la société de conversation sur le statut de ces œuvres.

Nous concentrerons notre étude sur la rue Mohamed Mahmoud en raison des liens qu'elle entretient avec les mondes numériques. De fait, presque tout autant que la place Tahrir, cette rue a fait l'objet d'une importante couverture médiatique et ce pour plusieurs raisons : tout d'abord, elle a été le théâtre de combats de rue meurtriers entre manifestants et forces de l'ordre en novembre 2011 ; par la suite, elle est devenue un véritable "sanctuaire d'écriture" ${ }^{4}$ puisque ses murs ont été investis par des artistes graffitis ou par des anonymes venus rendre hommage aux "martyrs" de la révolution égyptienne et à l'esprit de cette même révolution. L'effet plastique extraordinaire en plein cœur du centre-ville cairote de ces murs couverts de message a bien entendu attiré l'attention des médias, y compris des nouveaux médias. De fait, tout graffiti réalisé dans cette rue est immédiatement photographié, reproduit et discuté sur les réseaux sociaux, en particulier sur Facebook où de nombreux groupes ont été créés, y compris un groupe appelé "Rue Mohamed Mahmoud" ${ }^{5}$. Cela contribue à faire de cet endroit particulier de la géographie cairote un lieu à la fois physique et virtuel,

3. Sur ce point, voir, entre autre, Boraïe, S. 2012, Wall Talk, graffiti of the egyptian revolution, Le Caire, Zeitouna; Maslamani, M. 2013, Graffiti althawra al-masryia, Beyrouth, Arab Center for Research and Policy Studies; Abaza, M. 2012, An Emerging Memorial Space? In Praise of Mohammed Mahmud Street, article paru le 10 mars 2012, [http://www.jadaliyya.com/ pages/index/4625/an-emerging-memorial-space-in-praise-of-mohammedm] ; The Dramaturgy of a Street Corner, article publié le 25 janvier 2013, [http://www.jadaliyya.com/pages/index/9724/the-dramaturgy-of-a-streetcorner] ; Coletu, E. 2012, Visualizing revolution : the Politics of Paint in Tahrir, article publié le 18 avril 2012, http://www.jadaliyya.com/pages/index/5136/ visualizing-revolution_the-politics-of-paint-in-ta

4. Fraenkel, 2002.

5. "شارع محمد محمود " " " [https://www.facebook.com/st.martyrs?fref=ts]

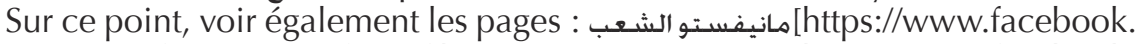

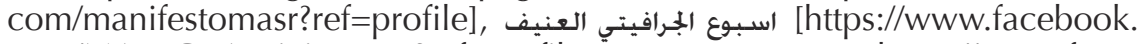

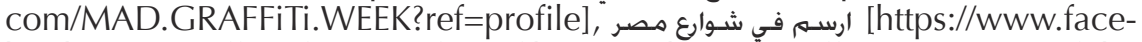
book.com/GraffitiOfEgypt?ref=profile], Revolution Graffiti - Street Art of the New Egypt [https://www.facebook.com/pages/Revolution-Graffiti-Street-Artof-the-New-Egypt/313913465299751] 
une porte d'entrée vers les mondes numériques. La rue Mohamed Mahmoud permet ainsi de mettre en évidence des caractéristiques qui sont inhérentes aux graffitis en général, mais extrêmement visibles dans cette configuration et que nous nous proposons d'explorer ici.

\section{MOHAMED MAHMOUD, PALIMPSESTE DE LA RÉVOLUTION ÉGYPTIENNE}

Le cas des graffitis égyptiens permet ainsi d'observer la façon dont la jeunesse arabe, "entrée sans crainte dans la société de conversation qu'a créé la toile universelle, a pu forger son propre langage" (GonzalezQuijano, 2012) et ainsi faire son entrée dans l'espace public égyptien. Remarquons dès à présent que par graffitis, nous entendons l'ensemble des propositions visuelles dialogiques présentes sur les murs de la rue $\mathrm{MM}$ : fresques (murals), pochoirs, gribouillages (kharabish) et inscriptions diverses. Les graffitis se présentent ainsi comme une forme d'écriture exposée déviante (Petrucci, 1980), mixte, utilisant à la fois le texte et l'image : ce sont des inscriptions dans l'espace public n'étant pas le fait des autorités et par conséquent possiblement illégales ${ }^{6}$. Par leur alliance de formes graphiques et de formes scripturales, les graffitis constituent d'une part un support visuel primordial d'intelligibilité du réel et, $\mathrm{d}^{\prime}$ autre part, se prêtent particulièrement bien à la démultiplication médiatique, sur Internet et ses réseaux sociaux.

Propositions visuelles dialogiques, les graffitis apparaissent comme une œuvre collective, perpétuellement inachevée, où chaque scripteur peut venir ajouter ou soustraire un élément de la configuration préexistante, dans la mesure où l'"œuvre" fait partie du domaine public. Nous avons utilisé le mot de palimpseste ${ }^{7}$ pour désigner ces processus d'écriture et réécriture continues à propos de la rue Mohamed Mahmoud. Régulièrement effacés, les graffitis réapparaissent immédiatement sur l'espace vierge du mur. Sur la photo ci-dessus, on peut distinguer les couches successives de graffitis qui se superposent les unes aux autres. Le fond rose, imitant le camouflage militaire, est l'œuvre de l'artiste égyptien Ammar Abo Bakr qui a recouvert l'entièreté du mur d'enceinte de I'AUC (American University in Cairo) dans la nuit précédant la commémoration de la bataille de la rue Mohamed Mahmoud, le 18 novembre 2013. Selon ses propres termes, il s'agissait de "recouvrir

6. Une loi est actuellement à l'étude en Egypte punissant les auteurs de graffitis "abusifs" dans l'espace public de peines allant jusqu'à quatre ans d'emprisonnement et 100.000 LE d'amende, loi qui confirme le caractère subversif de cette pratique "artistique".

7. Genette, 1982 ; Carle \& Huguet 2014. 


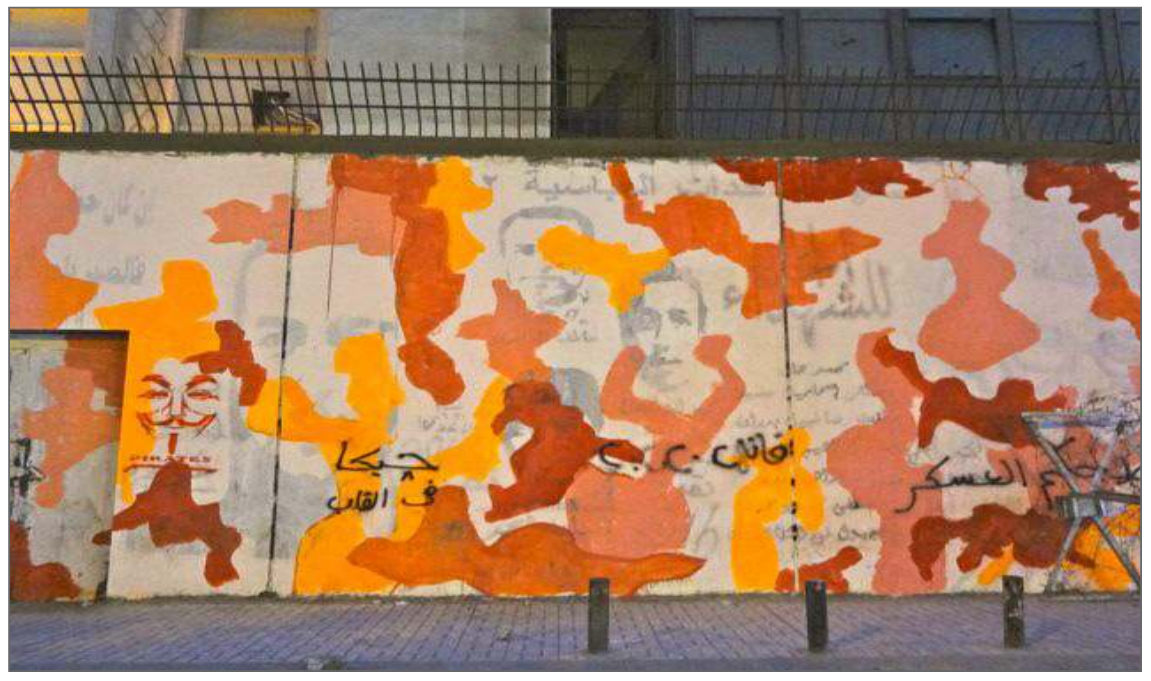

Rue Mohammed Mahmoud, novembre 2013, crédits photos : Zoé Carle.

pour devancer les autorités qui nettoient, donner aux gens un nouvel espace d'expression pour qu'ils écrivent ce qu'ils veulent" ${ }^{\prime \prime}$. Si dans ce cas l'effacement est une démarche artistique en elle-même, la plupart du temps il est plutôt le fait des autorités ${ }^{9}$ qui cherchent à reprendre le contrôle visuel de l'espace de parole et d'expression que constitue le mur. En dépit de ces tentatives de nettoyages, la multiplicité des scripteurs peut s'appréhender dans les couches successives de peinture et les anciens graffitis apparaissent sous la nouvelle couche de matière, comme on peut le voir sur cette photo. Le caractère polyphonique ou plutôt polygraphique de cet espace d'expression est immédiatement visible puisque sur le même mur cohabitent des dessins, des pochoirs, des inscriptions d'auteurs différents, qui se combinent ou se superposent. Sur l'espace du mur, un dialogue s'établit entre ces multiples traces écrites qui vont du slogan le plus simple aux réalisations artistiques les plus avancées.

8. Entretien avec Ammar Abo Bakr, Novembre 2013, Le Caire, Zoé Carle. 9. Sur ce point, voir Talon, C. 2012, Les effaceurs de rue du Caire, article publié le 24 septembre 2012 sur LeMonde.fr [http://www.lemonde.fr/afrique/ article/2012/09/24/les-effaceurs-des-rues-du-caire_1764076_3212.html] 


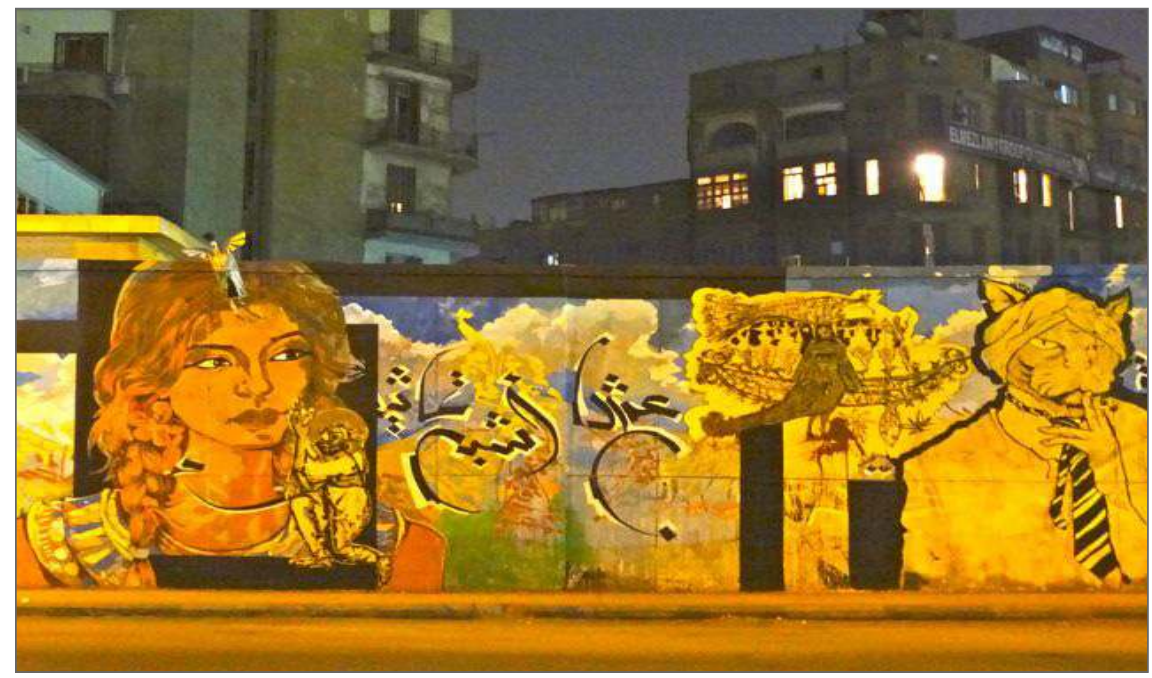

Rue Kasr El Nil ; juin 2013 ; auteurs : Ammar Abo Bakr et Ganzeer, crédits photos : Zoé Carle.

Toutefois, il serait réducteur de n'envisager ces graffitis que comme des objets artistiques, et c'est ce que montrent bien leurs prolongements sur la toile. L'hypothèse que nous avançons ici est qu'ils fonctionnent comme des médias, des propositions visuelles dialogiques, investies par des énonciateurs multiples intervenant au moment de la production et/ ou de la diffusion. Si d'une part l'auctorialité est sujette à caution dans la pratique du graffiti, y compris dans ses réalisations les plus artistiques ${ }^{10}$, d'autre part ce dialogisme fondamental est ce qui en fait un support médiatique particulièrement adapté à la "société de conversation"11. Par ailleurs, il est tout à fait remarquable que leurs logiques de création et de circulation soient étroitement liées aux mondes du numérique et aux réseaux sociaux. Les réseaux sociaux numériques constituent ainsi une source d'inspiration en amont de leur production mais ils constituent également une destination possible de ces graffitis au moment de leur archivage et de leur documentation. Ce qui est frappant, c'est qu'ils n'y sont pas uniquement conservés mais aussi et surtout discutés : de supports ils deviennent médias d'un débat public, portes-voix d'une nouvelle forme de parole politique contestataire. Le

10. À ce sujet, le grapheur Ammar Abo Bakr déclare «Je ne pourrai jamais dire qu'un graffiti cıest mon œuvre. (...) J'ai une idée ou un ami a une idée puis on en parle et je vais le faire. Mais au final, le graffiti n'est pas à moi, il est à tout les gens qui ont participé à l'élaboration de l'idée », Zoé Carle, entretien mené le 20 novembre 2013, Le Caire.

11. Gonzalez-Quijano, 2012. 
statut de ces médias graphiques in situ et ex situ permet également de se questionner plus largement sur l'utilisation de l'écriture et de ces productions artistiques comme voies d'influence des structures cognitives $^{12}$. En effet, si le développement des NTIC dans le monde arabe peut être envisagé comme un outil d'émancipation politique et sociale, il apparaît indispensable de penser les potentialités civiques de ces technologies à l'extérieur du cadre exclusif de la consommation ${ }^{13}$. Ces graffitis sont des jonctions entre utilisateurs connectés et ceux qui n'utilisent pas les nouveaux médias; ils font office de médiateurs entre usagers compétents des NTIC (les "arabités numériques" naissantes) qui utilisent les réseaux numériques pour regarder, lire, commenter et faire circuler les significations et contenus des graffitis mais ces derniers sont aussi des liens, des paroles adressées au quidam non connecté qui foule I'asphalte de la rue Mohammed Mahmoud et regarde, lit ces messages adressés aux passants dans un espace public non virtualisé. Il faudrait ici s'interroger plus longuement sur l'insertion de ces graffitis dans des processus de commoditization ${ }^{14}$, afin de voir s'ils changeront de statut. Pour le moment, étant donné leur statut de "moyens" au sens où ils sont le support d'un débat qu'ils contribuent à nourrir, et non pas de "fins" qu'ils pourraient être s'ils accédaient au statut d'œuvres par exemple; ils ne correspondent pas à des biens consommables, du moins pas dans leur ensemble ${ }^{15}$. Néanmoins, la question reste pertinente et indispensable à observer dans les années à venir.

Dans notre perspective, les graffitis sont pensés comme des "boîtes à outils", des "dispositifs" mettant en avant la participation des publics et posant frontalement la question de la propriété de l'espace public ${ }^{16}$.

12. Goody, 1979.

13. Proulx. \& Klein (dir.), 2012.

14. Adorno, 1938.

15. Sur ce point, voir Gonzalez-Quijano, Y., 2011, La révolution arabe, une création sans chef (d'œuvre) et dialogique, article publié le 13 décembre 2011 sur cpa.hypotheses.org [http://cpa.hypotheses.org/3152]

16. "J'appelle dispositif tout ce qui $a, d$ 'une manière ou d'une autre, la capacité de capturer, d'orienter, de déterminer, d'intercepter, de modeler, de contrôler et d'assurer les gestes, les conduites, les opinions et les discours des êtres vivants." Agamben, G. 2007, Qu'est-ce qu'un dispositif ?, Paris, Rivages poche. Sur la question de ce qui constitue un expace public, nous nous référons aux travaux de John Dewey, notamment Dewey, J. (trad. Joëlle Zask, 2003), Le Public et ses problèmes, Folio, coll. « Folio essais », Paris. 


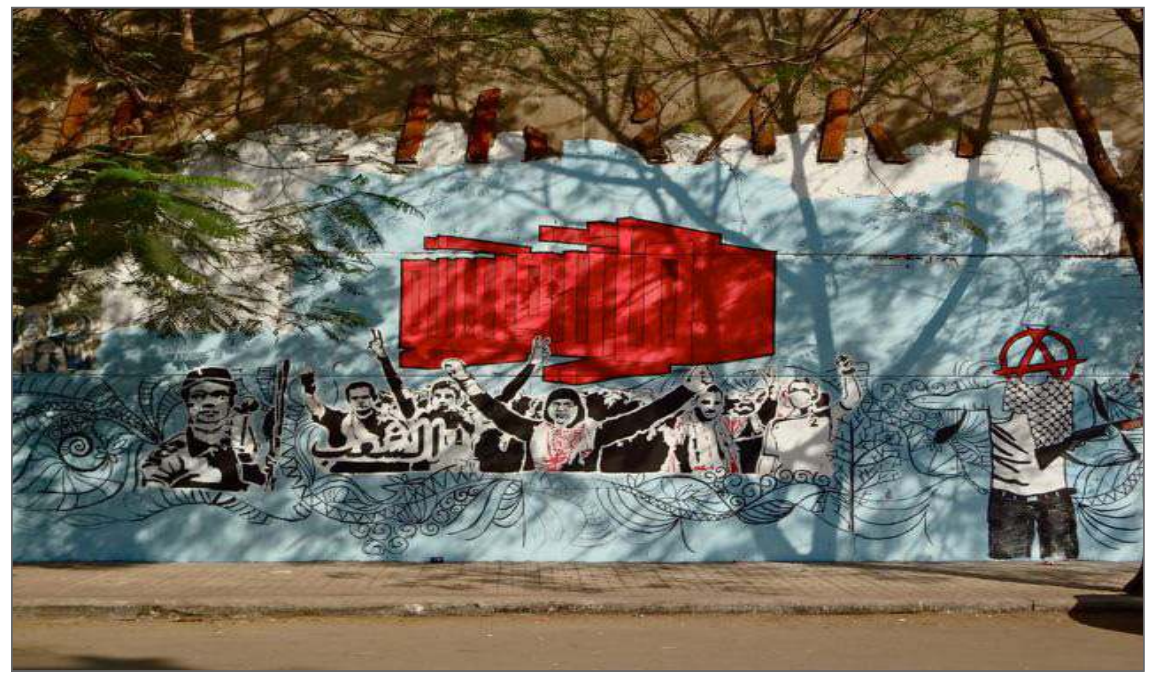

Rue Mohamed Mahmoud ; novembre 2013; "Manifeste du Peuple" ; auteurs : Kim, Ahmed Naguib, Tefa, crédits photos : Zoé Carle.

Ce graffiti, apparu il y a quelques mois dans la rue $M M$, a pour titre "Manifeste du peuple". On peut penser que ce manifeste, texte écrit à plusieurs, au nom d'un nous qui en l'occurrence est le "peuple", est le mur de la rue MM elle-même. Dans un geste autoréflexif, ce graffiti désigne l'espace d'écritures et de rencontres que constitue la pratique graphique commune du graffiti comme lieu d'expression populaire par excellence. Le mur devient ainsi manifeste, espace de revendication collective, où le "peuple" apparaît, avec toute la dimension fantasmatique que cela comporte. Le mot de "manifeste" suggère que ce mur est le lieu d'une participation collective, présente en acte dans les inscriptions de différentes mains, mais organisée dans un projet politique commun, ce qui ne va pas de soi. Il faut noter ici la grande performativité ${ }^{17}$ de ce graffiti

17. Définie par J.L. Austin dans ses cours de linguistique, la notion de performativité a opéré une migration depuis les disciplines linguistiques vers la sociologie et l'anthropologie. Désignant au départ des "actes de langage" c'est-à-dire des cas où le langage n'est pas utilisé à des fins descriptives ou constatives, mais pour réaliser des actes (promettre, ordonner, blasphémer, ...), elle a connu une certaine fortune en sciences sociales grâce aux travaux de Pierre Bourdieu, puis plus tard de Judith Butler, qui insistèrent sur I'aspect situationnel des énonciations et leurs enjeux institutionnels et sociaux. La notion de performativité réservée à des énonciations orales peut ainsi être utilisée pour décrire d'autres objets et notamment une performativité des actes écrits, comme a pu le démontrer I'anthropologue Béatrice Fraenkel (Fraenkel, 2006). C'est à ce titre que nous pouvons parler de performativité dans le cas de ce graffiti qui en nommant institue un objet, celui du manifeste populaire. 
qui instaure l'espace du mur comme un espace d'expression public et qui exhibe une caractéristique inhérente de la rue $M M$, cette énonciation collective par un sujet monumental et anonyme, désigné dans ce cas comme étant le "peuple". Cela rejoint les analyses de Béatrice Fraenkel sur les "écrits de New-York". Suite aux attentats du 11 septembre 2011, on a pu observer dans la ville de New-York le surgissement massif d'écritures publiques à visée mémorielle rendant hommage aux victimes tout autour du Ground Zero : "Les écritures exposées font saillir une autre dimension de l'espace urbain, sa dimension politique. L'espace public ainsi réaménagé montre à chacun qu'il appartient au même corps, à la même entité qui l'englobe, et chaque scripteur, chaque lecteur, parce qu'il produit cet espace, réaffirme sa qualité de citoyen. La nécessité d'écrire aux yeux de tous, et surtout la pulsion de signature qui s'empare de milliers de passants, évoque irrésistiblement l'idée d'une cérémonie civique, la scène improbable de la ratification du contrat social à l'origine de tous les contrats ${ }^{\prime 18}$.

Au même titre que pour les écrits de New-York de 2001, on observe en effet une sanctuarisation de la rue MM entre 2011 et 2013, qui devient un espace dédié d'écriture dans la géographie cairote et qui donne son sens social au rituel d'écriture. Toutefois, si le geste d'inscrire dans l'espace de la rue est en soi politique, la dimension politique des graffitis égyptiens est plus forte encore dans la mesure où leurs contenus eux-mêmes sont directement en prise avec les événements de l'actualité nationale. II ne s'agit pas uniquement de signer dans l'espace de la rue, mais de dénoncer ou de revendiquer, de rendre ainsi manifeste l'existence de ce collectif fantasmatique qu'est le peuple pour modifier le cours du destin national. Pour ce qui nous occupe ici, grâce à la diffusion sur ces autres murs que sont ceux des réseaux sociaux, il s'agit également d'élargir la portée de ces écrits en les reflétant sur le web, d'augmenter leurs significations et leurs forces de suggestion, en les faisant circuler sur les réseaux numériques, les rapprochant en quelque sorte de l'hypertexte utilisé dans des documents informatiques. Il est tout à fait intéressant de constater que le graffiti présent sur le cliché précédent représente en effet le logo d'une page Facebook où il est lui-même reproduit, ce qui accentue la liaison entre cette écriture in situ et son inspiration numérique, qui lui réserve également une place d'exposition privilégiée. ${ }^{19}$

18. Fraenkel, B., 2002.

19. Voir ici : [https://www.facebook.com/manifestomasr] Le graffiti a été réalisé en lien avec ce collectif, fondé en novembre 2013. 


\section{LE DOMAINE PUBLIC : GRAFFITIS ET INTERNET}

Loin du brouhaha d'une foule médiatique inaudible, le "numérique" qui nous intéresse ici, celui qui "palpe"20 et "chorégraphie"21 la réalité politique et culturelle d'un mouvement social est pour certains un objet qui pousse les murs et offre à l'individu d'affirmer sa singularité. En effet, depuis la fin de l'année 2010, I'usage des réseaux numériques et du web participatif à des fins de mobilisation sociale et de contestation des régimes arabes réactualise et réarticule I'observation des rapports entre technologies de communication et leurs capacités à provoquer des changements sociaux et politiques. Mais le type de média dont nous traitons ici, celui des graffitis et de leurs reflets sur le web participatif, ne doit pas être observé comme un objet mécanique et froid, ce qui rejetterait notre analyse du côté du positivisme mcluhanien. C'est bien "le médium lui-même" qui nous intéresse ici pour reprendre la citation qui ouvre cet article, ses effets sur l'individu ou sur la société et le changement d'échelle que produit chaque nouvelle technologie. Les usages de ce médium sont multiples, en fonction de l'utilisation qu'en font les populations qui reçoivent ces messages, avec leurs différentes stratégies de réception et d'appropriation qui révèlent différentes aptitudes et une habileté à composer avec eux. Au sein de la rue Mohammed Mahmoud et des murs qui la composent, comme une nécessité impérieuse, la parole est prise, volée, rendue, offerte; elle affirme, contre, argumente, illustre, critique, se trompe, se justifie, invente de nouvelles formes d'écritures. On ne s'attardera pas ici sur l'inventivité de l'arabzi ${ }^{22}$, objet de recherche en soi. Un nouveau récit fait son apparition simultanément dans la rue et sur les écrans des utilisateurs des réseaux sociaux qui suivent la révolution égyptienne. Apparaît alors le souci de documenter, de discuter, d'archiver, de devenir soi-même média puisque les médias traditionnels n'assurent plus leur mission de documentation du réel et offrent une image trop déformée de cette "révolution" aux contours flous, des actions qui sont menées en son nom et ne rendent pas compte de la façon nouvelle dont on échange sur les réseaux sociaux. ${ }^{23}$

20. Latour, 2012.

21. Gerbaudo, 2012.

22. Par arab-easy, arabic chat-alphabet, arabzi, arabish, nous entendons une forme d'arabe écrit composé à partir de caractères et de chiffres de l'alphabet latin. Encodage utilisé dès 2004 pour éditer le web 2.0 alors que des modules de saisies utilisant I'alphabet arabe n'existaient pas encore.

23. Cf. note de bas de page $n^{\circ} 2$. 
Dans ces reflets numériques, les usagers anonymes prennent pour étendard d'un "nous" plus ou moins bien défini les productions picturales et scripturales de la rue MM tout comme des images chocs des héros de la révolution, de martyrs, de policiers corrompus, etc. Sur la durée, les productions sont commentées, échangées, partagées sur des murs de profils personnels ou sur d'autres pages liées aux graffitis égyptiens. Elles glissent petit à petit vers le bas de l'écran suivant la logique de défilement vertical propre aux réseaux sociaux numériques (scroll down, scroll through). In situ, les murs s'effacent et se recouvrent d'autres graffitis mais, ex situ, sur les outils numériques, le web archive les conversations, les périodes de création, les tient encore ouvertes, garde les photographies de productions culturelles désormais recouvertes par d'autres coups de pinceaux ou de bombes de peinture. Les réseaux sociaux du numérique font discuter la mémoire de la rue MM et les messages dont elle se compose. Les différentes couches du palimpseste de cette rue apparaissent en filigrane grâce au reflet numérique de la rue contenu sur les réseaux sociaux.

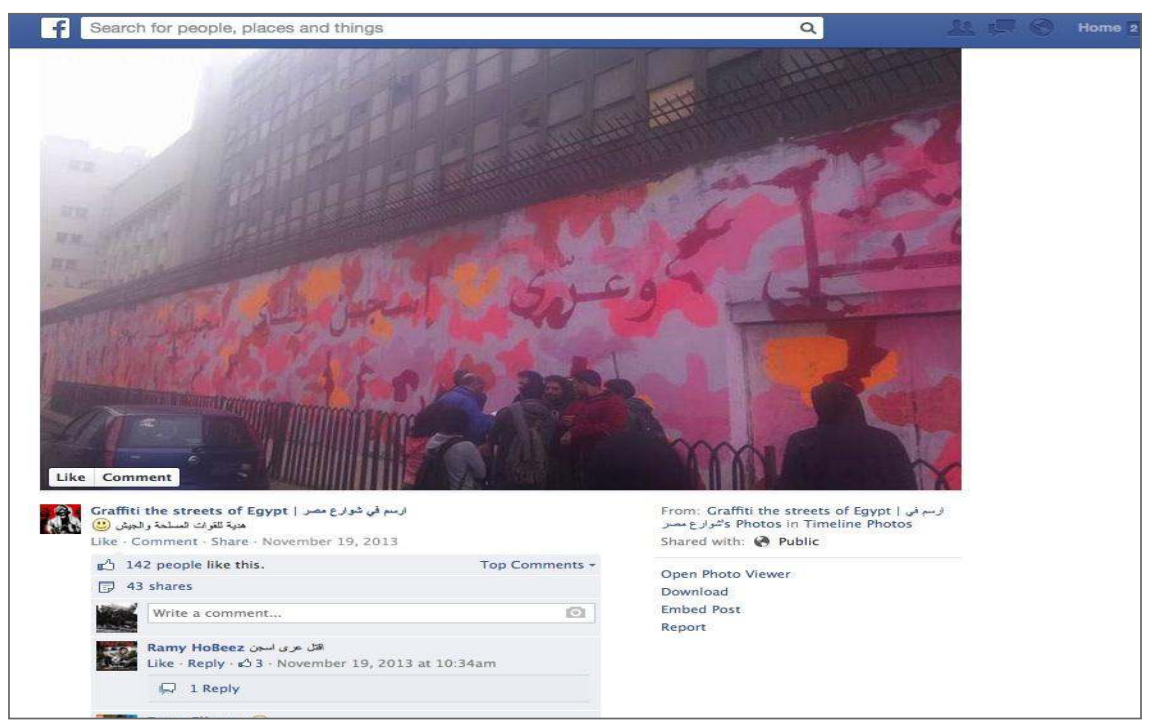

Capture d'écran du groupe Facebook

"Graffiti the streets of Egypt | ارسم في شوارع مصر" commentant la production de Ammar Abo Bakr réalisée en novembre 2013 sur les murs de la rue MM. 


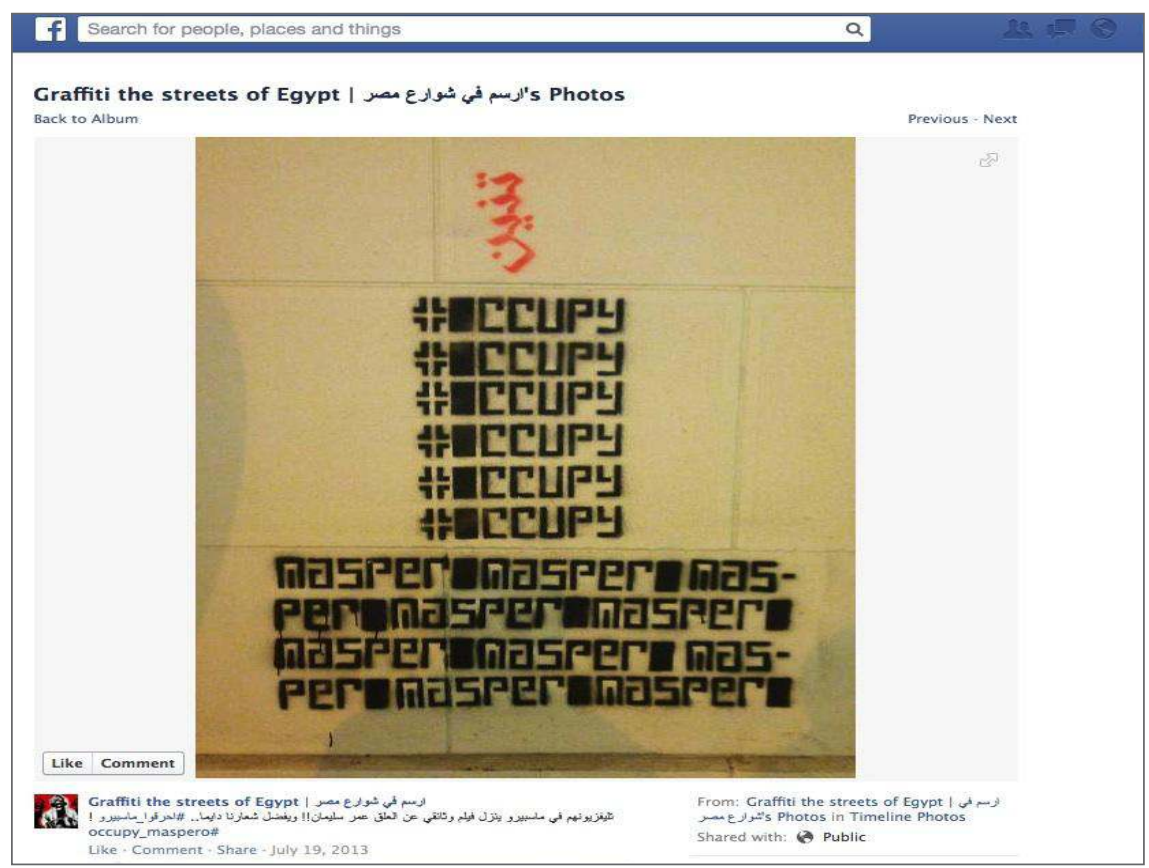

Capture d'écran du groupe Facebook

"Graffiti the streets of Egypt | ارسـم في شـوارع مصر "

commentant le pochoir "Occupy Maspero" appelant à l'occupation de la place Maspero (siège de la télévision et radio publique égyptienne)

et à la critique nécessaire des médias nationaux.

Sur ces deux plans (in et ex situ), un dialogue critique s'ouvre, amplifié par les technologies du numérique qui sont capables de capturer et de faire circuler des visions du monde. Nous notions précédemment la grande proximité de ces pratiques d'écritures avec la "société de conversation" mais les murs de la rue Mohammed Mahmoud et les peintures qu'ils contiennent sont aussi intrinsèquement liés à cette forme politique à part entière qu'est Internet ${ }^{24}$. Comme lors de ce mouvement de renaissance et d'inscription dans l'espace public d'une parole citoyenne critique envers le pouvoir débutée en 2010, Internet décloisonne les débats et les ouvre à de nouveaux participants mais renouvelle surtout les possibilités de critique et d'action. Nous avons noté le lien étroit qui existe entre cette rue et les mondes numériques, pour des raisons de pratique d'archivage et de circulation des graffitis, mais il faut noter surtout la proximité structurelle de ces deux éléments. Penser aujourd'hui les liens entre dispositifs de communication

24. Cardon, 2010. 
et mouvements sociaux dans le monde arabe est chose essentielle et cette réflexion ne peut s'effectuer qu'en portant un regard attentif sur

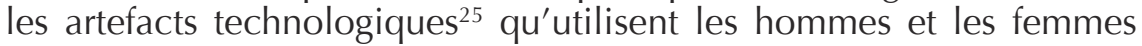
qui militent pour provoquer des changement au sein de leurs pays.

Ces artefacts et les assemblages qui en résultent dessinent une nouvelle forme d'espace de dialogue, d'espace public, qui, comme le remarque Richard Senett "peut être simplement défini comme un endroit où se rencontrent des étrangers" et où les hiérarchies s'effacent au profit d'un dialogue horizontal. Le palimpseste hypertextualisé que nous décrivons ici dessine cette nouvelle forme d'espace public d'échange et de communication capable de "montre(r) à chacun qu'il appartient au même corps, à la même entité qui l'englobe et que chaque scripteur, chaque lecteur, parce qu'il produit cet espace, réaffirme sa qualité de citoyen" ${ }^{26}$. Nous ne cherchons pas ici à exagérer le caractère inédit de ces propositions visuelles dialogiques puisque en effet les graffitis politiques sont une forme classique d'expression dans les moments de prise de parole collective. Néanmoins, le rôle joué par les médias sociaux et les réseaux numériques dans le devenir de la rue Mohammed Mahmoud est lui radicalement nouveau et est la résultante d'une évolution sur de nombreuses années qui compte pour principales étapes l'essor dans le monde arabe de l'imprimerie transnationale, suivi de celui de la télévision satellitaire et enfin, l'arrivée de I'Internet ${ }^{27}$.

Notre analyse permet d'affirmer que les images réelles et virtuelles des écritures exposées de la rue Mohammed Mahmoud sont à la fois le signe et le moyen d'un phénomène nouveau: la réappropriation progressive du lieu public égyptien par les citoyens, d'une part grâce aux mouvements sociaux et à l'occupation physique des lieux (dans les manifestations, à travers les graffitis et les écritures publiques), mais également grâce aux réseaux numériques. La rue $M M$ est à ce titre emblématique de cette prise de parole collective, un miroir tendu à un peuple égyptien aux contours indéterminés qui se réfléchit dans l'espace de la ville et dans ses débordements numériques. Ceux-là ne viennent pas constituer un espace public virtuel venant redoubler un espace public réel, mais au contraire ils sont les outils conjoints d'une réappropriation de celui-ci. Par ailleurs, les cultures numériques de la jeunesse égyptienne combinées à leurs pratiques scripturales et artistiques nous donnent à observer une modélisation particulière et

25. Ainsi que sur leurs fonctionnements et leurs architectures intrinsèques. Nous pensons en effet que faire des "digital humanities" aujourd'hui passe aussi par le fait de préter attention aux principes internes des services Internets et des outils numériques que l'on étudie.

26. Fraenkel, 2002.

27. Gonzalez-Quijano, 2012. 
nouvelle du concept de "village global ». Au sein de cette globalité, le public égyptien, à la fois auteur des scripts de la rue Mohammed Mahmoud, mais aussi lecteur et metteur en scène de leurs présences sur le web fait évoluer sa relation de spectateur passif vers le participant, créant ainsi des leviers d'empowerment citoyen.

Cette rue et son miroir sur le web s'incluent plus largement dans la naissance d'un "arabisme digital" évoqué précédemment où l'usage de nouveaux médias redessine des répertoires de communication nouveau. L'élément crucial de compréhension du rôle des médias sociaux au sein des mouvements sociopolitiques contemporains réside dans les interactions et les médiations que ces derniers établissent avec de nouvelles formes de publics. En effet, ils participent d'une part directement à la chorégraphie des manifestations, mais d'autre part sont partie prenant des processus de constitution symbolique d'un espace public, à la fois local et global grâce à l'universalisation que permet l'outil Internet ${ }^{28}$. Dans ces nouveaux processus, le dispositif de la rue MM ne constitue pas un outil de mobilisation direct mais s'intègre à ces chorégraphies de façon plus oblique, en tant que sanctuaire, à la fois in situ et ex situ, espace symbolique et artistique fournissant des références et un imaginaire révolutionnaire tout aussi indispensables aux mobilisations, et dans la constitution duquel les nouveaux médias et les réseaux numériques jouent un rôle fondamental. ${ }^{29}$

\section{GRAFFITIS ET DISPOSITIFS MÉDIATIQUES ALTERNATIFS}

Dans la constitution de cet imaginaire révolutionnaire, un certain nombre de thématiques sont privilégiées. Nous avons abordé rapidement la dimension funéraire de la rue $M M$ à travers la figuration des martyrs notamment dans le travail de I'artiste Ammar Abo Bakr, mais une autre dimension non moins importante est l'attention accordée au discours médiatique officiel et à la façon dont les médias font œuvre de propagande. Il s'agit là d'un thème central dans les différentes réalisations graphiques, qu'elles passent par de simples injures ou bien par des réalisations plus métaphoriques. Cette attention particulière portée au dispositif médiatique confirme le rôle particulier

28. Castells, 2012.

29. Il convient bien évidemment de nuancer ces analyses en remarquant que si elles sont valables du point de vue des pratiques scripturaires et de leurs intentions, il n'est pas certain qu'elles soient suivies d'effet dans la réception de ces graffitis, comme en témoigne la récente étude menée par Hannah El Ansary : http://www.jadaliyya.com/pages/index/19033/revolutionary-street-art_complicating-the-discours 
dévolu aux graffitis dans l'économie des discours et contre-discours révolutionnaires: ils s'affirment résolument comme un outil venant contester la prééminence d'un discours univoque dans un espace public égyptien où la liberté d'expression est encadrée par différents mécanismes de censure. Les graffitis, à leur façon et dans la mesure de leurs moyens, s'imposent ainsi comme une parole alternative, un média contestataire.

Si les graffitis entretiennent une relation particulière avec les nouveaux médias, il va de soi que le média graphique lui-même est l'un des plus anciens qui existe, comme le rappelle opportunément le grapheur Ganzeer $^{30}$ : "C'est le plus vieux des médias, avant même le papier. En tout cas, c'est un média différent, plus direct parce qu'il n'y a pas de rédacteur en chef ou d'éditeur par rapport aux médias traditionnels qui sont des médias plus élitistes." II est tout à fait intéressant que les activistes retrouvent ce moyen de communication à un moment de crise politique nationale où l'interprétation des événements est un enjeu majeur pour la maîtrise du discours officiel. De façon symptomatique, les graffitis se présentent comme une réaction populaire spontanée à un discours médiatique omniprésent et invasif, centralisé et contrôlé par les forces politiques ou pouvoir, tel que celui décrit par Ganzeer ; en d'autres termes, les graffitis offrent une alternative à un discours de propagande typique, et en ce sens nous pouvons parler de dispositifs médiatiques contestataires ${ }^{31}$. On observe en effet un discours conscient sur les médias dans la plupart des créations graphiques de la rue MM qui permet de considérer que ces médias se présentent avant tout comme des médias alternatifs. La dénonciation de la propagande officielle est en effet I'un des thèmes privilégiés de la rue $M M$, que cela soit par I'intermédiaire d'inscriptions toutes simples ("Media Whore" photo d'introduction), de pochoirs comme ceux du mouvement Kazeboon ${ }^{32}$, ou encore par des réalisations plus élaborées sur le plan plastique.

30. Extraits d'un entretien avec Ganzeer (décembre 2013, Le Caire, Zoé Carle).

31. Pour M. Linard, un dispositif “implique une mise en système délibérée des éléments et des conditions d'une action, une construction cognitive fonctionnelle, pratique et incarnée", in "Conception de dispositifs et changement de paradigme en formation", Education permanente, n 152, 2002 pp. 143-155.

32. Voir leur page Facebook: عسكر كاذبون [https://www.facebook. com/3askar.Kazeboon] 


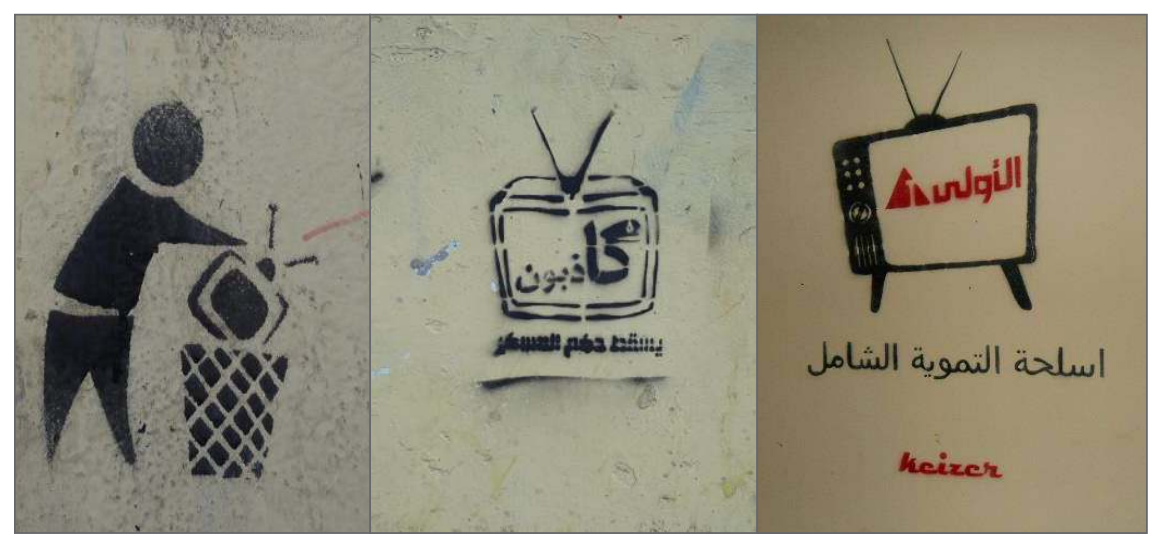

Collectif Kazeboon ; rue MM ; avril 2012 ; photo : Zoé Carle; Keizer ; mai 2013 ; "La une : arme d'enjolivement massif" [https://www.facebook.com/KeizerStreetArt?fref=ts]

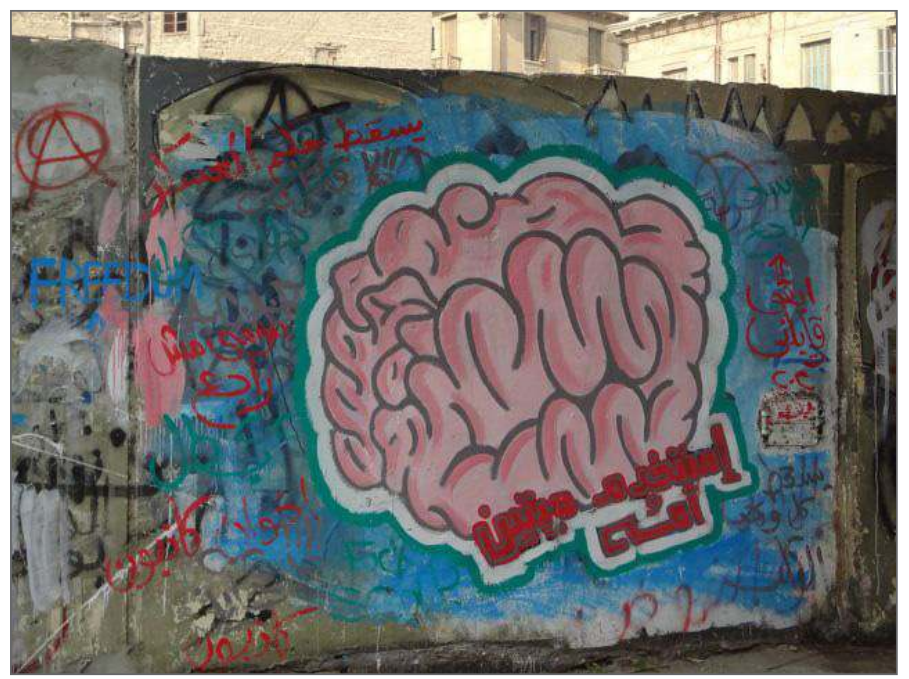

"Utilise ton cerveau" ; rue MM ; novembre 2013, crédits photo : Zoé Carle. 
L'abondance de mentions des médias traditionnels et des médias nationaux est particulièrement remarquable dans la rue $\mathrm{MM}$. Le thème principal étant bien sûr le lavage de cerveau (غسيل المخ en arabe) organisé par la propagande nationale qu'il s'agit de dénoncer pour la désamorcer. En ce sens, les auteurs de graffitis promeuvent un discours contestataire aux propriétés libératrices en désignant les canaux d'information traditionnels pour ce qu'ils sont, non le reflet de la réalité, mais un discours situé idéologiquement et comme tel sujet à caution.

L'injonction de ces graffitis est simple: le passant est invité à réfléchir au lieu de s'abandonner aveuglément à un discours officiel qui déforme voire falsifie les événements. Il s'agit d'une véritable guerre $d^{\prime}$ interprétation dans laquelle les graffitis prétendent jouer un rôle de révélateur et de contre-pouvoir.

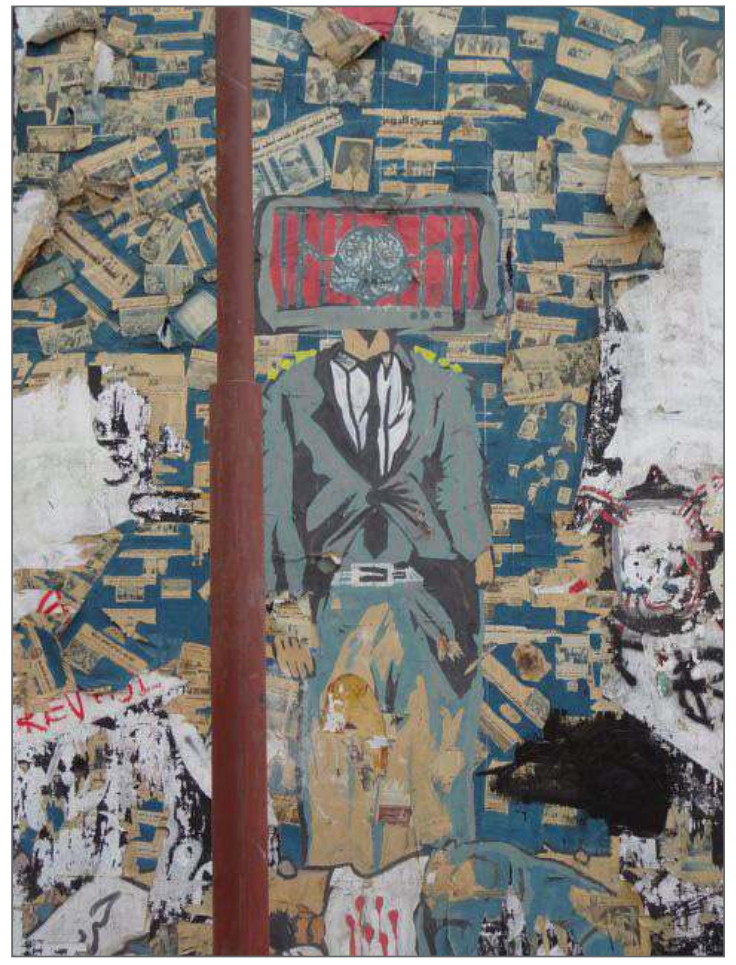

Rue MM ; novembre 2013 ; crédits photo : Zoé Carle.

Dans la photo ci-dessus, un personnage est figuré, le cerveau emprisonné dans un écran de télévision grillagé, alors que tout autour de lui sont collés des coupures de presse, extraites des grands quotidiens de la presse nationale comme Al-masry al-youm, Al-Watan, etc. L'utilisation de la technique du collage, associé à un mode de 
représentation plus métaphorique, est extrêmement efficace dans la perspective de dénonciation qui est celle du graffiti. Tous les médias sont concernés : télévisés, écrits, audio, tandis que la multiplicité des discours qui assaillent l'imaginaire et l'intelligence du spectateur sont figurés à travers la profusion de coupures de presse qui entourent le personnage.

D'innombrables graffitis abordent ce thème, montrant comment l'interprétation des événements devient une guerre dans laquelle les grapheurs, et plus largement les artistes, les journalistes citoyens et les activistes sont engagés, contre les médias traditionnels pour rétablir une vérité vacillante.

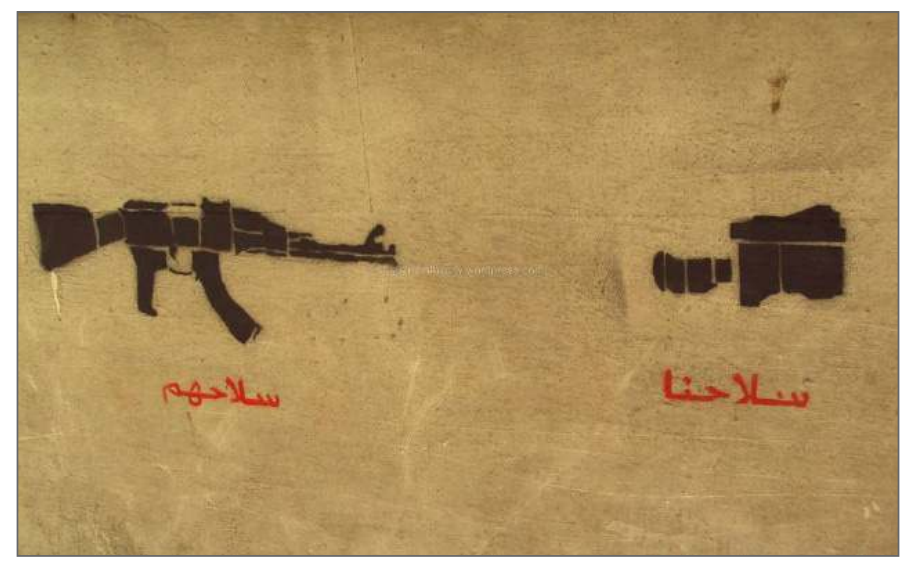

"Nos armes, leurs armes" ; juin 2011 ;

crédit photo : Suzee in the city (http://suzeeinthecity.wordpress.com)

Cette dernière image est très riche en ce qu'elle explicite le parallèle entre la guerre et les conflits d'interprétation médiatique. La fracture entre un "nous" et un "autre" hostile est mise en scène par la confrontation des deux objets et toutes les dénotations contradictoires qu'ils impliquent. L'image invite à un engagement fort du récepteur par la présence de ce "nous" ainsi qu'à une cohésion renouvelée d'un groupe mal défini mais dont l'énonciateur anonyme et le récepteur universel font radicalement partie. Par ailleurs, cette image opère une mise en abyme assez compliquée puisque ce qui est désigné ici c'est un outil médiatique et pas un graffiti, mais représenté dans un pochoir qui est destiné lui-même à être pris en photo et à circuler sur la toile. Cette image met ainsi en scène de façon subtile l'intrication étroite du support graffiti et des réseaux sociaux numériques que nous pouvons observer à d'autres échelles dans l'ensemble des graffitis de notre corpus. Elle 
illustre par ailleurs le lien qui peut exister entre ces murs et d'autres formes de médias et instaure un parallèle entre la guerre et les conflits d'interprétation : la bataille pour la mise en récit des événements se livre aussi rue $M M$, I'interprétation n'étant pas aussi irénique que la célébration constante $d^{\prime} u n$ " art révolutionnaire » peut le laisser penser. Dans un contexte politique troublé, l'enjeu médiatique n'est pas des moindres, et les auteurs de graffiti pourraient faire leur cette affirmation du poète salvadorien Roque Dalton : "La falsification de l'histoire de cette guerre est sa continuation par d'autres moyens." ${ }^{\prime 3}$

$S^{\prime}$ il n'est pas certain que les graffitis véhiculent un message positif s'opposant au discours officiel, - en effet, ils n'affirment rien - en revanche par leur structure et le dispositif qu'ils constituent, ils $s^{\prime}$ inscrivent contre les discours médiatiques traditionnels et en ce sens en sont un contre-pouvoir. C'est par conséquent ce qui permet de les considérer comme des dispositifs médiatiques alternatifs et populaires, que nous pourrions rapprocher du travail du collectif Kazeboon, du journalisme citoyen, et de cette volonté de faire soi-même média en utilisant les technologies numériques tout en restant ancré dans le réel des mouvements sociaux.

Il faut donc reconnaître le rôle particulier que jouent les graffitis dans l'appareil des discours médiatiques contemporains en Egypte et dans l'espace public en général. De façon évidente, il semble inévitable d'envisager ces "scripts" dans leur double dimension: physique et virtuelle, pour les considérer comme des médias dans le débat public. La similitude de structure entre ces propositions visuelles dialogiques et la façon dont s'organisent les relations dans les réseaux sociaux facilitent par ailleurs leur insertion dans l'espace numérique. Si la notion de "manifeste" semble être une caractérisation un peu trop précise pour désigner ce nouvel espace d'expression populaire, il n'en reste pas moins que ces œuvres collectives sont le symptôme et le signe d'un rapport renouvelé à l'espace public. Attaquant frontalement les discours médiatiques traditionnels, les graffitis deviennent des dispositifs de contestation efficaces, permettant une relation critique aux canaux de transmission plus classiques que sont la télévision, la radio et la presse. Au même titre que la littérature, ils invitent le spectateurlecteur à une mise à distance de l'actualité politique par leur mise en place de garde-fous interprétatifs et favorisent ainsi l'émergence d'une pensée herméneutique ne se contentant pas des évidences assénées par des médias omniprésents, mais cherchant au-delà des apparences à renouveler le regard posé sur l'actualité et la politique.

33. Dalton, 2005. 


\section{CONCLUSION}

Au delà des dispositifs de communication numériques et des médias classiques, force est de constater que "les révolutions se font avant tout par des citoyens hommes et femmes, qu'elles naissent à partir de faits ou d'évènements avec une forte charge symbolique" ${ }^{\prime \prime 4}$. Il s'avère également que les révolutions ou les soulèvements, peu importe les qualificatifs qu'on leur attribue, interviennent dans des contextes favorables à l'émergence d'une nouvelle vision du monde. Changer de point de vue sur la communication, les médias et les technologies qui s'y rapportent semble aujourd'hui plus que jamais nécessaire tout comme le fait d'élargir nos angles d'analyses sur la question des usages des $\mathrm{TIC}$ numériques à celle des pratiques communicationnelles globales. Les jonctions qui s'effectuent avec des phénomènes d'un autre ordre (et qui peuvent sembler, de premier abord, relever de pratiques plus anodines) avec de nouveaux dispositifs de communication bouleversent les modèles d'analyses que l'on pouvait auparavant plaquer sur des phénomènes socioculturels ou sociopolitiques. Néanmoins, il ne s'agit pas de tomber dans un enthousiaste naïf concernant I'usage des NTIC au sein des soulèvements arabes et plus précisément dans ceux qui concernent l'Égypte actuelle. À travers l'étude des créations figurants sur les murs d'une rue cairote in et ex situ, nous souhaitons réfléchir, dans une perspective communicationnelle et littéraire, à ce que signifie le surgissement d'écritures à la fois réelles et virtuelles lors d'un événement tel que la révolution égyptienne débutée en 2011 et dans un lieu tel que celui de la rue Mohammed Mahmoud. Ces écritures, ces scripts, ces productions artistiques laissent entrevoir à la fois de nouvelles stratégies culturelles et médiatiques des publics égyptiens et impliquent de nouvelles méthodologies de recherches pour appréhender certaines réalités socioculturelles et sociopolitiques de ces publics. II est certain qu'Internet augmente le nombre de locuteurs dans l'espace public, «en abaissant les contraintes traditionnelles qui étaient par exemple de donner son nom, de ne pas être péremptoire, de respecter l'autre dans un débat... On n'entre plus nécessairement dans l'espace public en empruntant les habits du citoyen dévoué à l'intérêt général, mais de façon plus subjective, avec des intérêts locaux, une voix singulière et des propos un peu froissés $»^{35}$; mais Internet bouleverse également la conception binaire que I'on pouvait se faire de l'espace public. Ces graffitis de la rue Mohammed Mahmoud et leurs augmentations numériques viennent nourrir un espace démocratique autre que celui de la compétition pour la représentation politique. Ils mettent à jour une voix, un « nous » et

34. Daghmi F., Toumi F., \& Amsidder A. (dir), 2013.

35. Cardon, 2010. 
une parole autre que celle qui apparaît sur les canaux d'informations classiques.

Les pratiques communicationnelles que nous analysons en nous attachant à étudier à la fois les productions en soi et leurs circulations sur les réseaux numériques nous font penser que les pratiques des arabités numériques actuelles ne doivent pas s'analyser uniquement en fonction de supports technologiques utilisés mais également en fonction d'actions communicationnelles conduites par des agents sociaux. Le changement de paradigme à l'œuvre dans la jonction graffiti-circulation sur le web (usage/TIC et pratiques/communication) est soutenu par la notion de dispositif : les dispositifs communicationnels de l'usager intègrent l'ensemble des TICN qu'il utilise d'une part, et sa façon de "faire dispositif" est une caractéristique des pratiques communicationnelles hypermodernes, d'autre part. 


\section{BIBLIOGRAPHIE}

AbazA, M., 2013, The Dramaturgy of a Street Corner, article paru le 25 janvier 2013 sur Jadaliyya.com [http://www.jadaliyya.com/pages/ index/9724/the-dramaturgy-of-a-street-corner]

AbazA, M. 2012, An Emerging Memorial Space? In Praise of Mohammed Mahmud Street, article paru le 10 mars 2012, sur Jadaliyya. com [http://www.jadaliyya.com/pages/index/4625/an-emergingmemorial-space-in-praise-of-mohammed-m]

Adorno, T., 2001, [1938], Le Caractère fétiche dans la musique, Paris, Allia, $96 \mathrm{p}$.

Agamben, G., 2007, Qu'est-ce qu'un dispositif ?, Rivages poche, 64 p.

Austin, J.L., 1962, "Performatif-Constatif ", La philosophie analytique, Cahiers de Royaumonts, Cahiers de Royaumont, Philosophie ${ }^{\circ}$ IV, Paris, Editions de Minuit, p. 271-304.

Austin, J.L. 1970, Quand dire, c'est faire (trad. G. Lane), Paris, Seuil, $208 \mathrm{p}$.

BASHÎR, M.J., 2011, Kitâb al-ultrâs. 3indamâ tata3ad\$a al-jamâhîr alTabî3a, Le Caire, Dâr Dawwin.

BECKER, H., 1988, [1982], Les mondes de l'art, Paris, Flammarion, 379 p.

Butler, J., 1990, Gender Trouble, New York, Routledge, 172 p.

CARdon, D., 2010, La démocratie Internet, Promesses et limites, La République des idées - Seuil, Paris, 101 p.

CARle Z. \& Huguet F., (à paraître), Les graffitis de la rue Mohamed Mahmoud, palimpseste de la révolution égyptienne, PURH, Rennes.

CAstells, M., 2012, " Ni dieu ni maître: les réseaux », FMSHWP-2012-02.

Coletu, E., 2012, Visualizing revolution : the Politics of Paint in Tahrir, article paru le 18 avril 2012 sur Jadaliyya.com [http://www.jadaliyya. com/pages/index/5136/visualizing-revolution_the-politics-of-paintin-ta]

Confavreux, J., 2012, Egypte: les voix confisquées, la parole libérée, article paru le lundi 25 juin 2012 sur Mediapart.fr [http://www. mediapart.fr/journal/international/220612/egypte-les-voixconfisquees-la-parole-liberee]

Daghmi, F., Toumi, F., \& Amsidder, A. (dir), 2013, Les médias font-ils les Révolutions? Regards critiques sur les soulèvements arabes, L'Harmattan, Paris.

Dalton, R. 2005, Les histoires interdites du Petit Poucet, Paris, L'Harmattan, $241 \mathrm{p}$.

Dupret B. \& FerRIE J.N. (dir.), 2008, Médias, guerres et identités. Les pratiques communicationnelles de l'appartenance politique, ethnique et religieuse, Paris, Editions des Archives contemporaines, 256 p.

El AnsARY H., 2014, Revolutionary Street Art: Complicating the Discourse, article paru le $1^{\text {er }}$ septembre 2014 sur Jadaliyya.com 
[http://www.jadaliyya.com/pages/index/19033/revolutionary-streetart_complicating-the-discours]

El Zein R. et Ortiz A. (dir., 2011), Sign of the Times, The popular Literature of Tahrir Protest Signs, Graffiti \&, Street Art in Shahadat, Testimonials to the changing landscapes of short form writing in the contemporary Middle East, New York, ArteEast.

Fraenkel B., 2002, Les écrits de septembre. New-York 2001, Editions Textuel, Paris, 159 p.

Fraenkel B., 2007, "Actes d'écriture: Quand écrire c'est faire", Langage \& Société n 121-122, sept-déc. 2007, pp. 101-112.

FraenKEL B., 2006, "Actes écrits, actes oraux: la performativité à l'épreuve de l'écriture » in "Performativité : Relectures et usages d'une notion frontière ", in Études de communication n ${ }^{\circ} 29$ (coord DENIS, J.), Lille, Université Charles-de-Gaulle-Lille 3, pp. 69-94.

Genette, G., 1982, Palimpsestes: La Littérature au second degré, Seuil, Paris.

Gerbaudo, P., 2012, Tweets and the Streets Social Media and Contemporary Activism, Pluto Press, Londres.

Gonzalez-Quijano, Y., 2011, "L'arabisme digital », Medium, $n^{\circ} 29$, Réseaux : après l'utopie, oct-nov-dec 2011, pp. 8-20.

Gonzalez-QuiJAno, Y., 2012, Arabités numériques Le printemps du Web arabe, Arles, Actes Sud-Sindbad, 187 p.

GonZalez-Quijano, Y., 2012, "La révolution arabe : une création sans chef (d'œuvre) et dialogique" sur Culture et politique arabes [http:// cpa.hypotheses.org/3152]

Gonzalez-Quijano, Y., GuaAybess, T. (dir.) 2009, Les Arabes parlent aux Arabes, la révolution de l'information dans le monde arabe, Arles, Actes Sud-Sindbad, 272 p.

Goody, J., 1979, La raison graphique, La domestication de la pensée sauvage, Editions de Minuit, Paris, $274 \mathrm{p}$.

Habermas J., 1962 [1978], L'espace public Archéologie de la publicité comme dimension constitutive de la société bourgeoise (trad. B. Launay), Paris, Payot, 325 p.

Holston, J., 1999, Cities and Citizenship, Durham, London, Duke University Press.

Huyghe F.B., MERZEAU L., 2012, "Réseaux : après l'utopie », Medium n 29, Réseaux : après l'utopie, oct-nov-déc 2011, pp. 4-5.

Jeanneret Y., 2008, Penser la trivialité. Volume 1: La vie triviale des êtres culturels Paris, Éd. Hermès-Lavoisier, coll. Communication, médiation et construits sociaux, $267 \mathrm{p}$.

JeANNERET Y., 2007, Y-a-t-il (vraiment) des technologies de l'information ? Villeneuve d'Ascq, Presses Universitaires du Septentrion, coll. "Savoirs mieux ", $134 \mathrm{p}$.

Latour, B., 2012, Enquête sur les modes d'existence, La Découverte, Paris, 498 p. 
Laroussi F., Lienard, F., 2012, "Des écrans à la rue : I'émergence de communautés sociolinguistiques virtuelles pendant la révolution tunisienne », dans Usages et pratiques des publics dans les pays du Sud - Des médias classiques aux TIC, AMsidDer, A., DaGHMI, F. ET Touml, F. (dir.). Agadir : Université Ibn Zohr, pp. 289-297.

Mc Luhan, M., 1977 [1964], Pour comprendre les médias, Seuil, Paris.

Petruccl, A., 1993, Jeux de lettre - Formes et usages de l'inscription en Italie $-11^{\mathrm{e}}-20^{\mathrm{e}}$ siècles, Editions de l'EHESS, Paris, $271 \mathrm{p}$.

Pierandrel, E., 2012, Urban Cairo. La primavera araba dei graffiti, Informant Ebook Quotidiani [http://inform-ant.com/it/ebook/ urban-cairo.-la-primavera-araba-dei-graffiti et sa carte des graffitis égyptiens: http://www.inform-ant.it/mappa-graffiti-cairo.html]

ProulX, S. \& KleIN A., dir. 2012, Connexions. Communication numérique et lien social, Presses universitaires de Namur, Namur, 353 p.

Sennet, R., 2010, "The Public Realm" in The Blackwell City Reader, Second Edition, edited by Gary Bridge \& Sophie Watson, John Wiley \& Sons, Hoboken, pp. 261-271.

Souchier E., Jeanneret Y. 1999, Pour une pratique de l'écrit d'écran, Xoana 6, p. 98-99.

SOUCHIER E., 1998, L'image du texte, pour une théorie de l'énonciation éditoriale, Cahiers de médiologie, $\mathrm{n}^{\circ}$ 6, p. 137-145.

SOOKE, A., 2013, Egypt's powerful street art packs a punch, article publié le 9 mai 2013 sur bbc.com [http://www.bbc.com/culture/ story/20130508-egypts-street-art-revolution/]

Talon, C., 2012, Les effaceurs de rue du Caire, article publié le 24 septembre 2012 sur LeMonde.fr [http://www.lemonde.fr/afrique/ article/2012/09/24/les-effaceurs-des-rues-du-caire_1764076_3212. html] 